\title{
TRAIL receptor-mediated JNK activation and Bim phosphorylation critically regulate Fas-mediated liver damage and lethality
}

Nadia Corazza, ${ }^{1}$ Sabine Jakob, ${ }^{1}$ Corinne Schaer, ${ }^{1}$ Steffen Frese, ${ }^{2}$ Adrian Keogh, ${ }^{3}$ Deborah Stroka, ${ }^{3}$ Daniela Kassahn, ${ }^{1}$ Ralph Torgler, ${ }^{4}$ Christoph Mueller, ${ }^{1}$ Pascal Schneider, ${ }^{4}$ and Thomas Brunner ${ }^{1}$

${ }^{1}$ Division of Immunopathology, Institute of Pathology, University of Bern, Bern, Switzerland. ²Division of General Thoracic Surgery and 3Division of Visceral and Transplantation Surgery, Department of Clinical Research, Insel University Hospital, Bern, Switzerland. ${ }^{4}$ Department of Biochemistry, University of Lausanne, Lausanne, Switzerland.

\begin{abstract}
TNF-related apoptosis-inducing ligand (TRAIL) is a member of the TNF family with potent apoptosis-inducing properties in tumor cells. In particular, TRAIL strongly synergizes with conventional chemotherapeutic drugs to induce tumor cell death. Thus, TRAIL has been proposed as a promising future cancer therapy. Little, however, is known regarding what the role of TRAIL is in normal untransformed cells and whether therapeutic administration of TRAIL, alone or in combination with other apoptotic triggers, may cause tissue damage. In this study, we investigated the role of TRAIL in Fas-induced (CD95/Apo-1-induced) hepatocyte apoptosis and liver damage. While TRAIL alone failed to induce apoptosis in isolated murine hepatocytes, it strongly amplified Fas-induced cell death. Importantly, endogenous TRAIL was found to critically regulate anti-Fas antibody-induced hepatocyte apoptosis, liver damage, and associated lethality in vivo. TRAIL enhanced antiFas-induced hepatocyte apoptosis through the activation of JNK and its downstream substrate, the proapoptotic Bcl-2 homolog Bim. Consistently, TRAIL- and Bim-deficient mice and wild-type mice treated with a JNK inhibitor were protected against anti-Fas-induced liver damage. We conclude that TRAIL and Bim are important response modifiers of hepatocyte apoptosis and identify liver damage and lethality as a possible risk of TRAIL-based tumor therapy.
\end{abstract}

\section{Introduction}

Induction of apoptotic cell death is a major goal of modern anticancer therapy $(1,2)$. However, different types of tumors show unequal sensitivity to different chemotherapeutic drugs or have acquired resistance to these apoptotic triggers. Thus, additional and alternative treatments are needed for successful therapy of distinct types of tumors. TNF-related apoptosis-inducing ligand (TRAIL) is a member of the TNF family with potent apoptosisinducing properties in tumor cells (3-5). Unlike its relatives TNF- $\alpha$ and FasL, which also induce potent proinflammatory responses or promote apoptosis in a variety of nontransformed tissue cells, TRAIL appears to preferentially induce cell death in tumor cells while leaving normal tissue cells unaffected $(5,6)$. Furthermore, TRAIL strongly synergizes with conventional chemotherapeutic drugs in inducing tumor cell apoptosis, making it a most promising candidate for future cancer therapy $(6,7)$.

While the signaling pathways leading to tumor cell apoptosis have been extensively investigated, relatively little is currently know about the role of TRAIL in normal untransformed tissue cells. In particular, it is at present unclear whether therapeutic administration of TRAIL, alone or in combination with other apoptotic triggers, may cause severe tissue damage or even death. For example, injection of an agonistic anti-Fas (CD95) antibody into mice promotes rapid hepatocyte apoptosis, liver damage, and death

Nonstandard abbreviations used: AST, aspartate transaminase; Bim $\mathrm{EL}_{\mathrm{L}}, 23-\mathrm{kDa}$ band of Bim; RIP, receptor-interacting protein; TRAIL, TNF-related apoptosisinducing ligand.

Conflict of interest: The authors have declared that no conflict of interest exists. Citation for this article: J. Clin. Invest. 116:2493-2499 (2006). doi:10.1172/JCI27726.
(8). In marked contrast, in vivo administration of recombinant TRAIL has been found to induce apoptosis only in transplanted human tumors while causing no detectable harm in recipient liver cells (5). Recently, however, the restriction of TRAIL-induced cell death induction to tumor cells has been challenged. Jo and colleagues found that recombinant TRAIL induces massive apoptosis in isolated human hepatocytes (9), identifying untransformed hepatocytes as potential victims and hepatitis as a potential side effect of TRAIL therapy. Similarly, Chen and colleagues reported that endogenous TRAIL is involved in liver damage during experimentally induced hepatitis (10). This observation is of particular interest as other members of the TNF family, i.e., FasL and TNF- $\alpha$, have also been implicated in the pathogenesis of hepatitis (11-13), suggesting that TRAIL may act synergistically with other apoptosis triggers in promoting hepatocyte death. Hence, the role of TRAIL in liver cell apoptosis is currently far from being fully understood.

In this study, we therefore investigated the role of TRAIL in Fas-induced hepatocyte apoptosis and associated lethality. FasL is expressed by activated cytotoxic T cells and NK cells (14), and Fas-induced target cell apoptosis and tissue damage have been implicated in a variety of immunopathologies, including graftversus-host disease $(15)$ and hepatitis $(11,13)$. Interestingly, we found that recombinant TRAIL alone failed to cause apoptosis in isolated murine hepatocytes but strongly enhanced FasL-induced cell death. In vivo, TRAIL appears to be a critical response modifier of hepatocyte death, as TRAIL-deficient mice were significantly more resistant to agonistic anti-Fas antibody-mediated hepatocyte apoptosis, liver damage, and associated lethality. We further demonstrated that TRAIL-induced amplification of Fas-induced liver damage required JNK activation and phosphorylation of its 
A

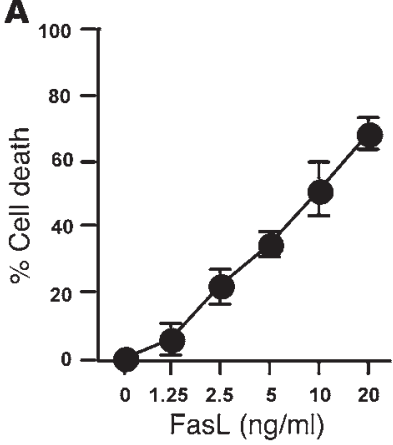

B
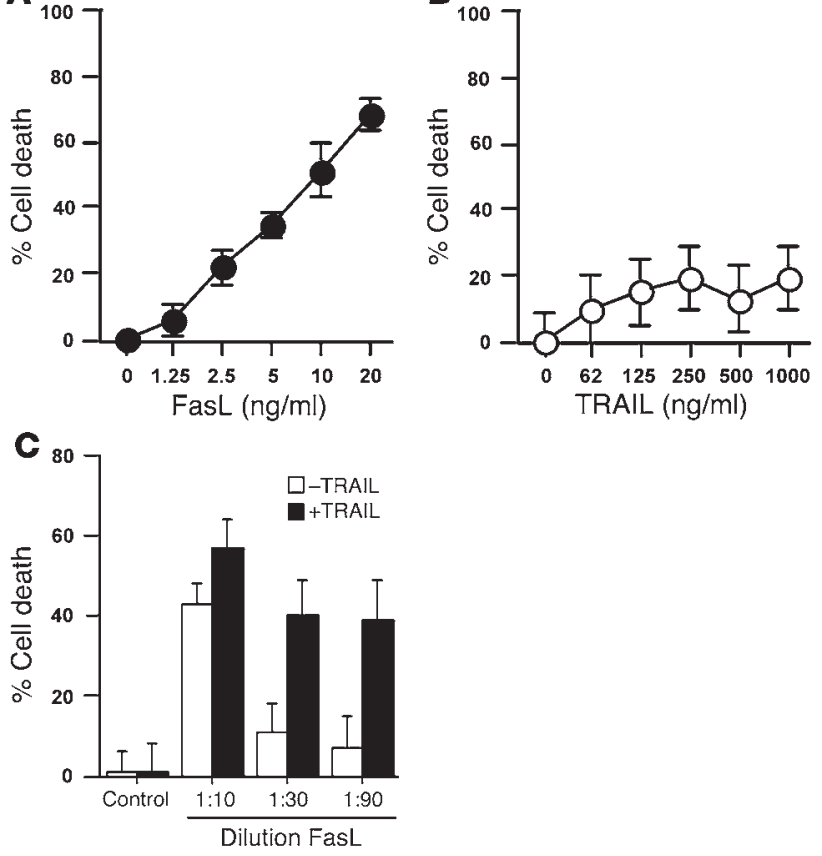

Figure 1

TRAIL amplifies FasL-induced hepatocyte apoptosis in vitro. Isolated murine hepatocytes were treated with increasing concentrations of soluble Fc-FasL (A) or TRAIL (B) or pretreated with or without TRAIL $(30 \mathrm{ng} / \mathrm{ml})$ prior to induction of apoptosis with serial dilutions of soluble FasL from Neuro-2 cells (C). Mean values of quadruplicates \pm SD of 1 representative experiment out of 3 are shown.

downstream target and proapoptotic Bcl-2 homolog Bim. Our study identifies TRAIL and Bim as important response modifiers of hepatocyte apoptosis and liver damage as a potential risk of TRAIL-based antitumor therapy.

\section{Results}

TRAIL enhances Fas-induced hepatocyte apoptosis in vitro. TRAIL was previously found to lack apoptosis-inducing property in isolated murine hepatocytes in vitro (16). In marked contrast, TRAIL appears to play a critical role in experimentally induced hepatitis in vivo (10). As these data suggest that TRAIL alone fails to induce apoptosis but may modulate other triggers of hepatocyte apoptosis, we tested the effect of TRAIL on Fas-induced hepatocyte death in vitro. As reported previously (16), we found that TRAIL alone did not induce a substantial increase in apoptosis in isolated murine hepatocytes exposed to a wide range of TRAIL concentrations (Figure 1, B and C). As expected, soluble FasL induced hepatocyte apoptosis in a dose-dependent manner (Figure 1A). Surprisingly, however, while TRAIL alone was an ineffective apoptosis trigger, it synergistically enhanced FasL-induced hepatocyte cell death (Figure 1C). Similar results were also obtained in isolated human hepatocytes (data not shown).

Endogenous TRAIL is critical for anti-Fas-induced liver damage. As TRAIL enhanced Fas-induced hepatocyte apoptosis in vitro, we further explored this issue in vivo. The injection of the agonistic anti-Fas antibody (Jo2), initially characterized by Nagata and colleagues (8), is a useful model for in vivo studies of Fas-induced signaling events in the liver. Injection of anti-Fas antibody leads to rapid hepatocyte apoptosis, liver damage, and associated lethality (8). In order to study a potential involvement of endogenous TRAIL in this signaling process, livers of wild-type and TRAILdeficient mice were analyzed 4 hours after administration of a lethal dose of anti-Fas antibody. Whereas livers of wild-type mice were hemorrhagic and necrotic, TRAIL-deficient livers displayed only minimal macroscopic signs of injury (Figure 2A). Consistent with this observation, the level of liver aspartate transaminase (AST) in the serum of TRAIL-deficient mice was 5 times lower than that monitored in wild-type mice (Figure 2B), and histological examination of TRAIL-deficient livers revealed a grossly intact architecture with minimal areas of tissue damage (Figure 2C). This was in sharp contrast with wild-type livers, in which signs of massive hepatocyte apoptosis and large areas of hemorrhagic necrosis were evident (Figure 2C). This observation is unlikely to be strongly dependent on a specific genetic background, as similar results were obtained in $\mathrm{C} 57 \mathrm{BL} / 6$ and Balb/c mice (Figure 2B and data not shown).

A hallmark of anti-Fas-induced apoptosis in liver cells is the activation of caspase 3 , a feature that was readily demonstrated by immunostaining for the cleaved, active form of caspase 3 , which stained positive in the vast majority of wild-type hepatocytes but in few TRAIL-deficient hepatocytes (Figure 2D). These data were corroborated by Western blot analysis. Partial processing of the 32-kDa procaspase 3 to the larger $18-\mathrm{kDa}$ caspase subunit was detected in wild-type, but not TRAIL-deficient, liver extracts (Figure $2 \mathrm{E}$ ). Taken together, these data indicate that caspase 3 activation, tissue damage, and hemorrhagic necrosis induced by antiFas antibodies in the liver are all greatly enhanced in the presence of endogenous TRAIL.

TRAIL accelerates anti-Fas-induced lethality. In agreement with the observation that TRAIL-deficient mice showed reduced caspase activation and liver damage, we further found that TRAIL-deficient mice were more resistant than wild-type mice to anti-Fas-induced death, although this protection was incomplete. Four hours after anti-Fas administration, $86 \%$ (6 of 7) of wild-type, but only $14 \%$ ( 1 of 7) of the TRAIL-deficient mice had died. For some TRAILdeficient mice, death was only delayed by $1-2$ hours, but $43 \%$ of TRAIL-deficient mice survived over 24 hours (Figure 3). These data suggest that TRAIL facilitates Fas-induced liver damage and thereby enhances Fas-induced lethality.

TRAIL is required for anti-Fas-induced JNK activation. As TRAIL failed to directly induce apoptosis in isolated hepatocytes but enhanced Fas-mediated cell death in vitro and in vivo, we hypothesized that TRAIL receptor engagement may activate a noncaspase pathway. Various reports have shown that all death receptors can, in addition to mediating Fas-associated death domain (FADD) recruitment and caspase 8 activation, trigger JNK activation via the receptor-interacting protein (RIP) $(17,18)$. We therefore determined whether JNK is differentially activated in hepatocytes from wild-type or TRAIL-deficient mice after anti-Fas antibody injection. There was indeed a time-dependent increase in the levels of phosphorylated JNK (reflecting JNK activation) in liver extracts from anti-Fas-injected wild-type mice (Figure 4A). In marked contrast, JNK was only minimally activated in the liver of TRAIL-deficient mice although total expression levels of JNK remained comparable. The rapid induction of JNK activation (Figure 4A) in liver samples from wild-type but not TRAIL-deficient mice correlated with the enhanced processing of procaspase 3 (Figure $4 \mathrm{~A}$ ) and the increase in serum transaminase levels (Figure 4B). 


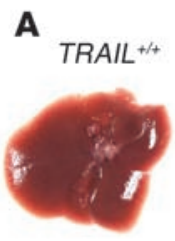

Control
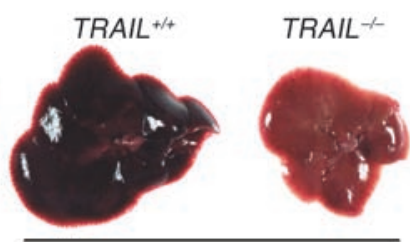

Anti-Fas
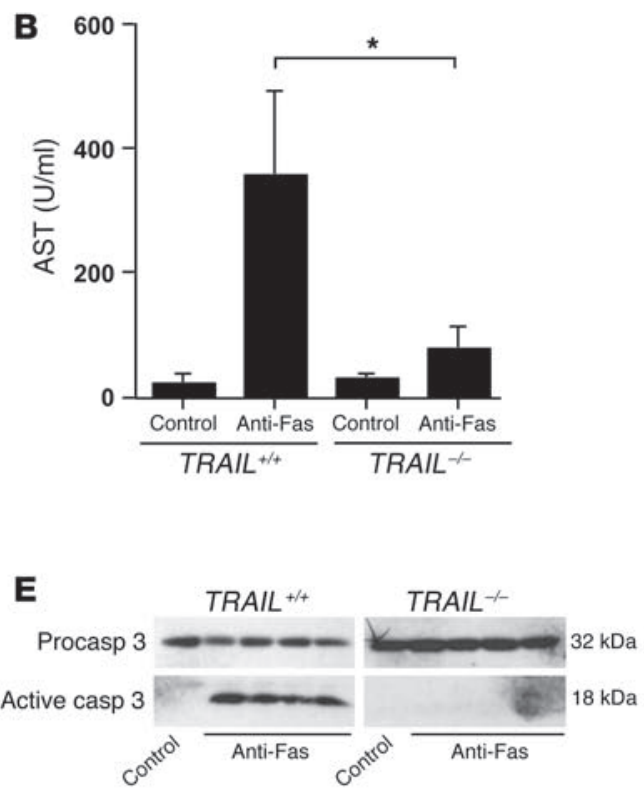

C $H \& E$

TRAIL $^{+/ 4}$

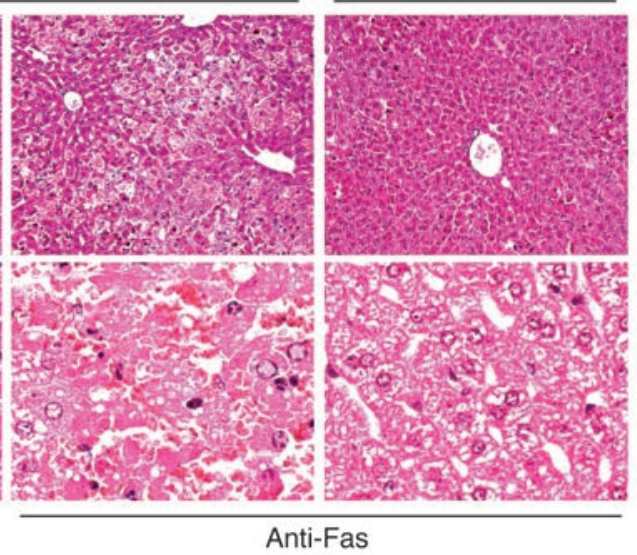

D

Casp 3

TRAIL ${ }^{+/+}$

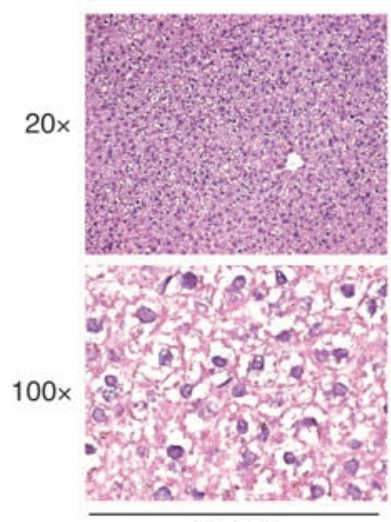

Control

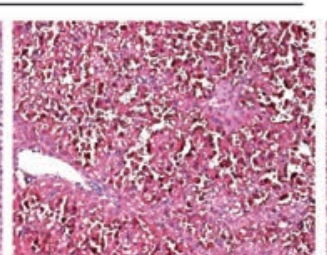

TRAIL ${ }^{-1}$

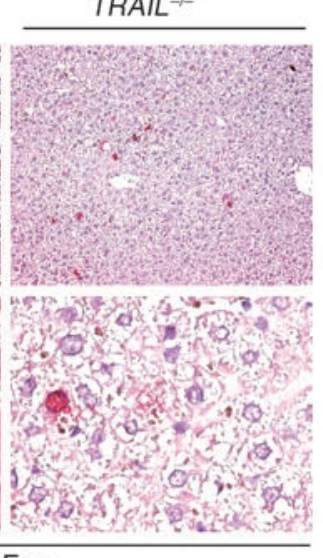

\section{Figure 2}

Attenuated Fas-induced liver damage in TRAIL-deficient mice. Wild-type or TRAIL-deficient mice were injected i.v. with PBS as a control or anti-Fas antibody. Liver tissue and serum samples of mice still alive after 4 hours were isolated and analyzed. (A) Macroscopic analysis of intact liver from untreated wild-type $\left(T R A / L^{+/+}\right)$mice and anti-Fas-injected wild-type and TRAIL-deficient $\left(T R A / L^{-/}\right)$mice. The liver of antiFas-treated wild-type mice is hemorrhagic and necrotic. (B) Serum levels of liver transaminase (AST) in control (PBS) or anti-Fas-treated wild-type or TRAIL-deficient mice. One representative experiment out of 3 is shown. $n=4$ mice per group, mean \pm SD. ${ }^{*} P<0.01$, Student's $t$ test. (C) Histological analysis of liver sections from untreated wild-type (TRAIL+/+, control), anti-Fas-treated wild-type, and $T R A I L-d e f i c i e n t$ mice. Low- and high-power magnification of representative samples is shown. (D) Immunohistochemistry for active caspase 3 (Casp 3). Caspase 3-positive apoptotic cells are stained in red. (E) Detection of caspase 3 activation in control-treated or anti-Fas-treated liver samples from wild-type or TRAIL-deficient mice by Western blot. The 18-kDa fragment indicates caspase cleavage and activation. Samples from 1 control and 4 anti-Fas-treated mice are shown.

JNK activation is critical for Fas-induced liver damage. To investigate whether JNK activation is required for anti-Fas-induced liver damage, we treated wild-type animals with control or the JNK inhibitor SP600125 (19) prior to injection with the anti-Fas antibody. Reduced levels of serum transaminases were monitored in JNK inhibitortreated mice versus control (Figure 4C). In agreement with this finding, TRAIL-mediated enhancement of FasL-induced apoptosis was attenuated in isolated murine hepatocytes after treatment with JNK inhibitor (data not shown). This supports the notion that JNK activation by TRAIL is required for Fas-induced liver cell apoptosis.

TRAIL- and JNK-dependent phosphorylation of Bim during Fas-induced liver damage. In addition to phosphorylating c-Jun and thereby activating the transcription factor AP-1, JNK also phosphorylates other targets. One of them is the proapoptotic Bcl-2 family member Bim (20). After JNK-induced phosphorylation, Bim, which is usually sequestered by microtubules (21), translocates to the outer mitochondrial membrane, where it promotes the release of cytochrome $c$ and thereby induces the activation of caspases $(20,22)$. Interestingly, Bim is involved in a positive amplification loop, as phosphorylated Bim can be cleaved by caspase 3 to yield a more potent inducer of cytochrome $c$ release (22). Thus, similarly to Bid, the proapoptotic activity of Bim is potentiated upon cleavage by caspases. We found that, upon anti-Fas-induced liver damage, the $23-\mathrm{kDa}$ band of Bim $\left(\mathrm{Bim}_{\mathrm{EL}}\right)$ was rapidly converted into 2 slower migrating species (Figure 5A). Interestingly, this change in Bim migration was strongly attenuated in samples from TRAIL-deficient mice. Previous reports have shown that these higher molecular weight bands represent hyperphosphorylated forms of BimeL $(20,22)$, which was confirmed by dephosphorylation of protein samples with calf intestinal alkaline phosphatase (Figure 5B) (22).

To confirm a direct connection between JNK activation and Bim phosphorylation, we assessed the effect of the JNK inhibitor 


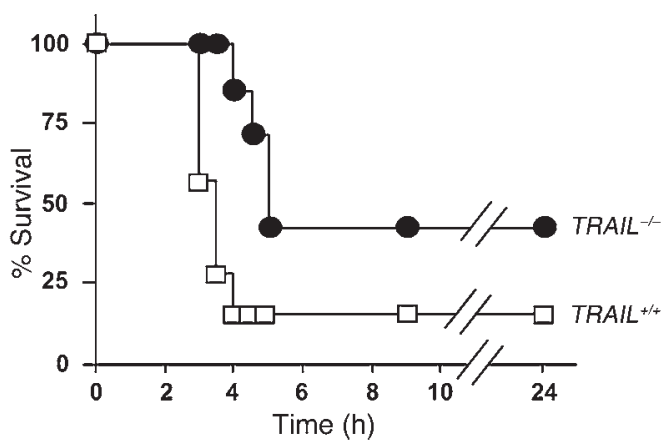

Figure 3

TRAIL accelerates anti-Fas-induced lethality. Survival curve of antiFas-treated wild-type $\left(T R A I L^{+/+}\right)$and TRAIL-deficient $\left(T R A I L^{-/-}\right)$mice over 24 hours. One representative experiment out of 2 is shown. $n=7$ mice per group.

SP600125 on anti-Fas-induced BimeL hyperphosphorylation in vivo. JNK inhibitor reduced BimeL phosphorylation and stabilized its hypophosphorylated form in liver samples from JNK inhibitor-treated animals. This reduced phosphorylation of Bim $\mathrm{BL}_{\mathrm{EL}}$ was paralleled by lower caspase 3 activation (Figure 5C).

Bim is required for anti-Fas-induced liver damage. These data suggested that TRAIL-induced JNK activation and Bim EL $_{\text {phosphor- }}$ ylation may be important in the regulation of Fas-induced liver damage. To test the involvement of Bim in Fas-induced hepatocyte apoptosis, $\mathrm{Bim}^{+/-}$or $\mathrm{Bim}^{-/-}$mice (23) were administered anti-Fas antibody. In the absence of a single Bim allele, a significant reduction of liver damage and release of liver transaminases occurred, an effect that was further enhanced by the complete loss of Bim (Figure 6A). Thus, Bim deficiency protected mice from anti-Fasinduced liver damage, as did the absence of TRAIL. In agreement with a linear pathway of TRAIL and Bim activation, we did not observe further protection against Fas-induced liver damage in the simultaneous absence of TRAIL and Bim (Figure 6A). The protection from Fas-induced apoptosis in Bim-deficient hepatocytes was further confirmed by a reduced histological damage (Figure 6B) and in situ activation of caspase 3 (Figure 6C). This correlated with increased resistance of Bim-deficient hepatocytes to Fas-induced apoptosis in vitro and with the inability of TRAIL to enhance Fasinduced apoptosis in the absence of Bim (Figure 6D).

To rule out the possibility that differential expression of signaling molecules of the canonical Fas signaling pathway may have accounted for the reduced anti-Fas-induced liver damage in TRAIL-deficient

\section{Figure 4}

Role of JNK in TRAIL-mediated sensitization of Fas-induced apoptosis. (A) Wild-type (TRAIL $\left.{ }^{+/+}\right)$or TRAIL-deficient mice (TRAIL ${ }^{-/-}$) were injected with PBS or anti-Fas antibody and euthanized at indicated time points. Liver protein samples were analyzed for phospho-JNK (p-JNK), active caspase 3, and JNK as loading control. Two mice per group are shown. (B) Serum transaminase levels (AST) were analyzed in wild-type and TRAIL-deficient mice at indicated time points after anti-Fas injection. Mean values \pm SD of 3 mice per group are shown. (C) Wild-type mice were injected i.p. with control or JNK inhibitor SP600125 (inh) prior to injection of anti-Fas antibody. Serum transaminase levels (AST) were analyzed after 4 hours. Bars represent mean values \pm SD of 7 mice per group. Statistical differences were analyzed by Student's $t$ test. ${ }^{*} P<0.01$. and Bim-deficient mice, we analyzed the expression levels of Fas, caspase 8 , Bid, and caspase 3 by Western blot. Figure 6E shows that liver expression levels of these proteins were almost identical in wild-type and mutant mice. Similarly, comparable levels of Bim were found in wild-type and TRAIL-deficient mice.

\section{Discussion}

TRAIL is a potent inducer of apoptosis in transformed and tumor cells (3), and its synergistic action with various chemotherapeutic agents (7) makes it an attractive candidate for anticancer therapy. However, the outcome of TRAIL exposure in untransformed cells is currently less understood and is controversial (24), and our data support the notion that liver damage is one of the potential risks of TRAIL-based antitumor therapy. Previous reports have shown that TRAIL may induce apoptosis in human hepatocytes (9), a finding that was not confirmed by others (16). Zheng et al. have recently shown that lectin-induced hepatitis, which requires the activation of liver lymphocytes, is strongly attenuated in TRAIL-deficient mice (ref. 10 and N. Corazza and T. Brunner, unpublished observations). This suggests that, depending on the inflammatory context or the presence of synergistic apoptosis triggers, TRAIL may be a potent inducer (or modifier) of hepatocyte apoptosis. Lectininduced hepatitis, however, was previously reported to critically depend also on the presence of FasL (25). Our findings provide direct evidence for the combined action of TRAIL and FasL in liver damage, in which TRAIL regulates Fas-induced apoptosis through JNK and Bim. While TNF- $\alpha$, FasL, and TRAIL can all induce JNK activation via a RIP-dependent pathway $(17,18)$, this is the first description, to our knowledge, of the involvement of Bim downstream of TRAIL-induced JNK activation and of its role in regulating Fas-induced caspase activation.

Korsmeyer and colleagues showed that cleavage and activation of Bid by caspase 8 is required for Fas-induced apoptosis in type II cells, such as hepatocytes (26). Similarly, we demonstrate here that Bim is also crucial for the execution of Fas-induced hepatocyte

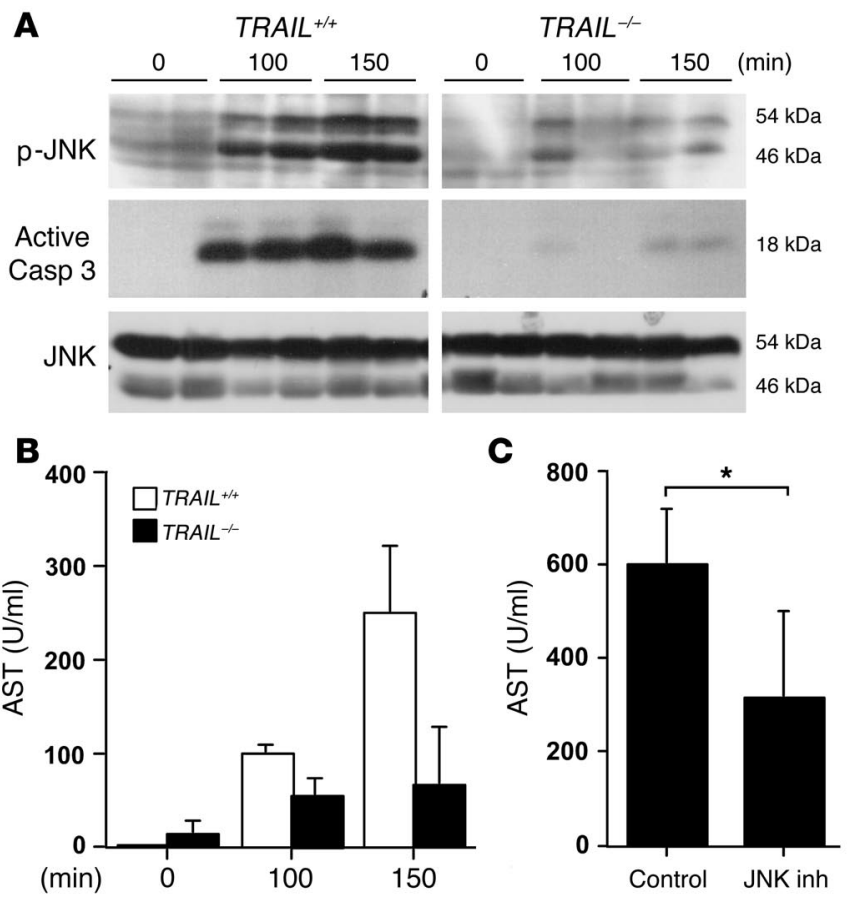


A
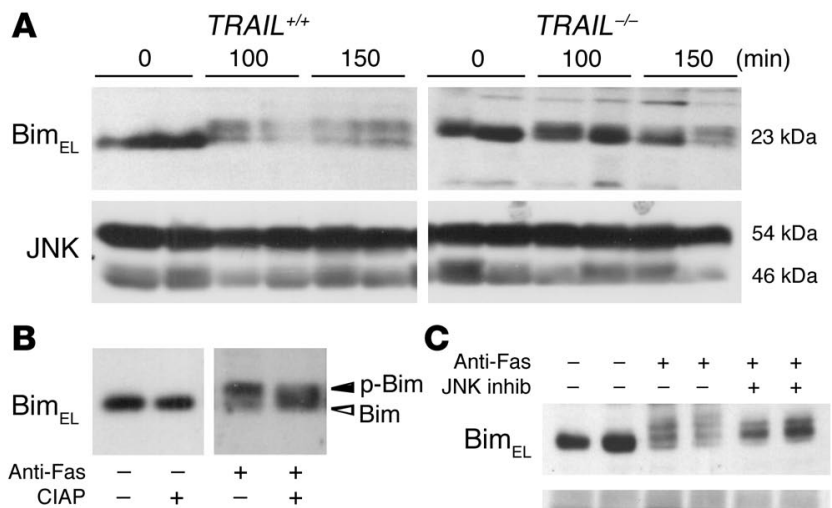

C

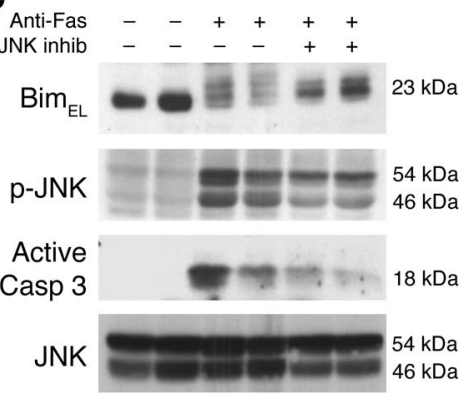

\section{Figure 5}

Role of Bim in TRAIL-mediated sensitization of Fas-induced apoptosis. (A) Wild-type or TRAIL-deficient mice were treated as in Figure 4A (identical samples), and liver protein samples were analyzed for Bim $\mathrm{EL}_{\mathrm{L}}$ and JNK as loading control. (B) Dephosphorylation of BimeL by calf intestinal phosphatase (CIAP). Liver protein samples from control or anti-Fas-injected mice were treated with or without calf intestinal phosphatase, and Bim $\mathrm{EL}$ was detected by Western blot. (C) Wild-type mice were treated with JNK inhibitor and anti-Fas as in Figure 4B, and liver protein samples were analyzed by Western blot for $\mathrm{Bim}_{\mathrm{EL}}, \mathrm{p}$-JNK, active caspase 3 , and JNK as loading control. Two mice per group are shown. $n=7$ per group. apoptosis. Thus, we propose that Bim represents a second mitochondrial amplification loop, which further amplifies caspase 8and Bid-initiated caspase activation. Bid is cleaved by caspase 8 (27, 28), promoting the release of cytochrome $c$ from the mitochondria and subsequent apoptosome formation. However, truncated Bid may initially only be able to induce release of little cytochrome $c$ and to activate low levels of caspase 9 and 3, which may, however, be sufficient to selectively cleave and activate phosphorylated but not unphosphorylated BimeL (22), initiating amplifying cycles of cytochrome $c$ release, apoptosome formation, and caspase activation. Alternatively, TRAIL/JNK- and caspase 3-activated Bim $\mathrm{EL}_{\mathrm{L}}$ may promote the release of Smac/Diablo, thereby releasing the brake by neutralizing caspase inhibitors, such as XIAP (29). While the exact mechanism of Bid and Bim $\mathrm{EL}_{\mathrm{L}}$ cooperation in Fas-induced mitochondrial apoptosis remains to be elucidated, our data support an important role for TRAIL, JNK, and Bim $\mathrm{EL}_{\mathrm{L}}$ in enhancing Fas-induced hepatocyte apoptosis. TRAIL-mediated modulation of apoptosis is, however, likely not limited to type II Fas signaling in hepatocytes, but may extend to other apoptosis signals proceeding via the mitochondria. It is interesting that TRAIL-deficient thymocytes show increased resistance to a variety of intrinsic apoptosis triggers, including UV and $\gamma$ irradiation, glucocorticoids, and $\mathrm{T}$ cell receptor ligation $(24,30)$. TRAIL signaling may therefore represent a general regulatory mechanism of the mitochondrial apoptosis pathway in different cell types.

Finally, one particular aspect of TRAIL-mediated enhancement of Fas-induced liver cell apoptosis still needs to be better understood. The relevant source of endogenous TRAIL in the liver is still uncertain. Previous reports have shown that liver-homing NK and NKT cells constitutively express TRAIL, which is crucial for the control of liver metastasis (31-33). Thus, liver-homing lymphocytes are a likely source of TRAIL in the experimental setup described in this study. Importantly, the cellular composition of intrahepatic leukocytes in wild-type and TRAIL-deficient mice is indistinguishable (data not shown). Alternatively, it is possible that hepatocytes express autologous TRAIL, as recently described in chimpanzees and humans (34); however, as the hepatitis-enhancing activity of TRAIL is confined to intrahepatic lymphocytes and not to stromal cells, it is more likely that TRAIL is derived from cells of hematopoietic origin (10). In addition, immunoglobulin $(\mathrm{Fc})$ receptors are required for anti-Fas-induced liver damage (35), and it is therefore likely that TRAIL- and Fc receptor-expressing cells are identical and that anti-Fas-induced $F c$ receptor activation may induce TRAIL cell surface expression in these cells.

Our data strongly support the notion that liver damage and possible death represent potential risks of TRAIL-based antitumor therapies in human patients. FasL is readily expressed in intrahepatic lymphocytes and macrophages during inflammatory responses and may synergize with TRAIL in promoting devastating liver damage. Furthermore, it can currently not be excluded that combination therapy with TRAIL and other apoptosis triggers may also result in increased apoptosis of untransformed cells, i.e., as we have observed in murine thymocytes (30). A better understanding of the TRAIL receptor-initiated signaling pathways in tumor and normal cell and of their interaction with other apoptosis pathways may help to develop successful TRAIL-based tumor therapies while avoiding devastating and potentially lethal side effects.

\section{Methods}

Induction of hepatitis. Young adult TRAIL-deficient (32) and Bim-deficient mice (23) (kindly provided by Immunex, Seattle, Washington, USA, and A. Strasser, Melbourne, Victoria, Australia, respectively) (all backcrossed for more than 6 or 10 generations, respectively, to the C57BL/ 6 background), TRAIL $\times$ Bim double-deficient mice (generated by breeding TRAIL-deficient with Bim-deficient mice), and wild-type C57BL/6 mice were injected i.v. with anti-Fas antibody (Clone Jo2; BD Bioscience - Pharmingen) at a dose of $0.25 \mu \mathrm{g} / \mathrm{g}$ of body weight. In some experiments, mice were pretreated 30 minutes before anti-Fas administration with the JNK inhibitor SP600125 (19) by i.p. injection $(25 \mu \mathrm{g} / \mathrm{g}$ of body weight). Animals were either observed for 24 hours (survival experiments) or euthanized at matched time points, and serum and liver samples were harvested. Serum AST levels were measured using a commercially available kit (505-OP; Sigma-Aldrich). Selected experiments were also performed using wild-type and TRAIL-deficient $\mathrm{Balb} / \mathrm{c}$ mice (backcrossed for 10 generations to the Balb/c background). All animal experiments were reviewed and approved by the Animal Experimentation Review Board of the State of Bern.

In vitro cell death assay. Murine hepatocytes were isolated by perfusion of liver lobes with $\mathrm{Ca}^{2+}$ - and $\mathrm{Mg}^{2+}$-free HEPES buffer, followed by HEPES buf- 


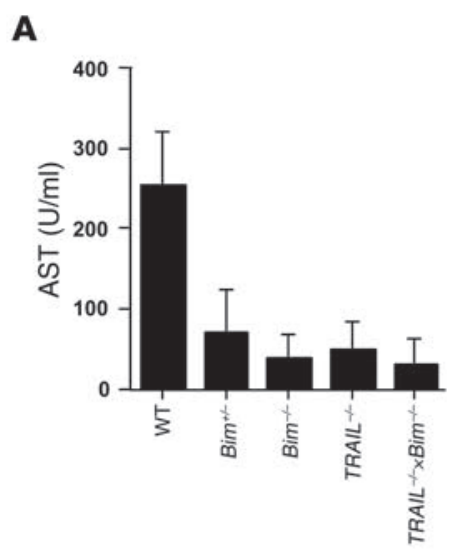

B $\mathrm{H} \& \mathrm{E}$

$\mathrm{Bim}^{+/+}$

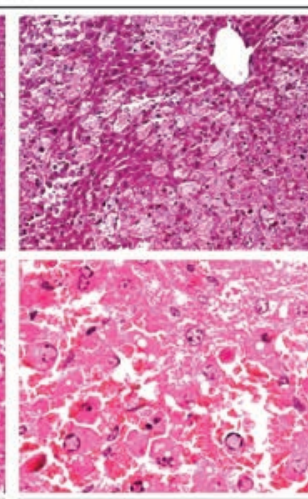

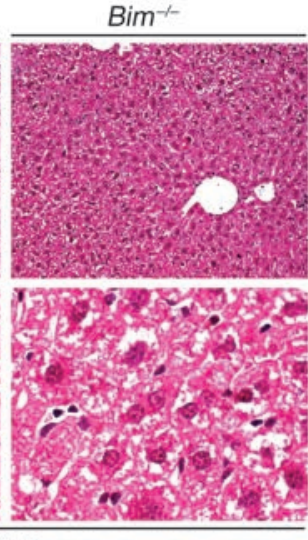

Anti-Fas
D

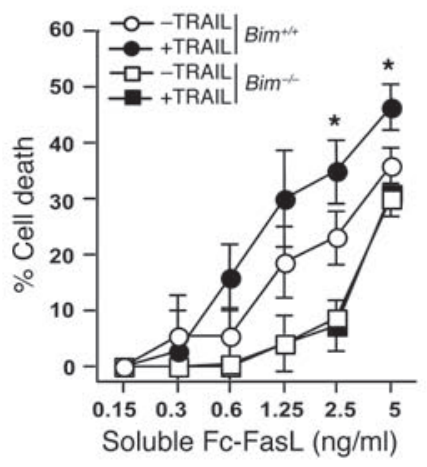

E

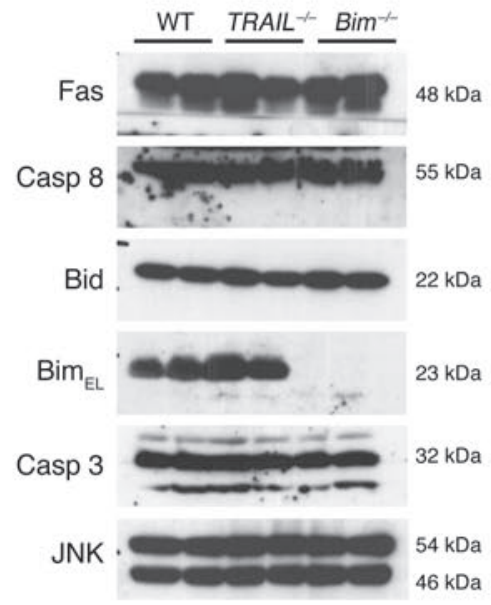

C Casp 3

Control

$\mathrm{Bim}^{+/+}$

20x
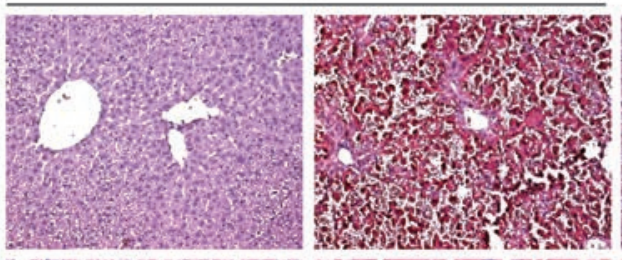

$\mathrm{Bim}^{-1}$

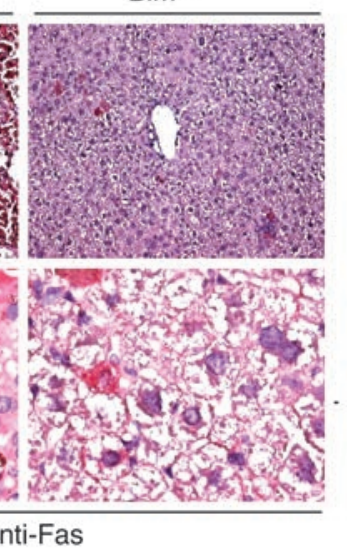

\section{Figure 6}

Bim is required for anti-Fas-induced liver damage. (A) Wild-type, Bim heterozygous $\left(\mathrm{Bim}^{+/-}\right)$, Bim homozygous-deficient (Bim $\left.{ }^{--}\right)$, TRAIL-deficient (TRAIL--) , and TRAIL $\times$Bim doubledeficient mice (TRAIL $\left.{ }^{--} \times \mathrm{Bim}^{--}\right)$were injected with anti-Fas antibody, and serum transaminase levels (AST) were analyzed after 4 hours. Mean values \pm SD of 4 mice per group are shown. (B) Histological analysis of liver sections from untreated wild-type mice $\left(\mathrm{Bim}^{+++}\right)$or anti-Fas-treated wild-type and Bim-deficient mice $\left(\mathrm{Bim}^{--}\right)$. Low- and high-power magnification of representative samples is shown. (C) Immunohistochemical detection of active caspase 3 (apoptotic cells, red) in control-treated wild-type mice $\left(\mathrm{Bim}^{+/+}\right)$or anti-Fas-treated wild-type or Bim-deficient mice $\left(\mathrm{Bim}^{-/}\right)$. (D) Hepatocytes isolated from wild-type $\left(\mathrm{Bim}^{+/+}\right)$ and Bim-deficient $\left(\mathrm{Bim}^{--}\right)$mice were pretreated with buffer control or $30 \mathrm{ng} / \mathrm{ml}$ TRAIL prior to apoptosis induction with increasing concentrations of soluble Fc-FasL. Mean values \pm SD of quadruplicates of 1 representative experiment out of 3 are shown. ${ }^{\star} P<0.05$, Student's $t$ test, $\mathrm{Bim}^{+/+}$with or without TRAIL. (E) Western blot analysis of liver extracts from wild-type, TRAIL-deficient (TRAIL $L^{--}$), and Bim-deficient (Bim-l-) mice (2 mice per group). Expression levels of Fas, caspase 8, Bid, Bim $\mathrm{EL}$, caspase 3, and JNK as loading control are shown.

fer containing $0.5 \mathrm{mg} / \mathrm{ml}$ collagenase IV and $5 \mathrm{mM} \mathrm{CaCl}_{2}$. After physical dissociation, viable cells were isolated by a $45 \%$ Percoll density gradient. Isolated hepatocytes were cultured in William's E medium (Sigma-Aldrich) containing $10 \%$ FCS. After overnight adherence, medium was changed and cells were treated with recombinant hTRAIL (untagged form; R\&D Systems) and soluble FasL (culture supernatant of FasL-transfected Neuro-2 cells, ref. 36; or soluble Fc-FasL fusion protein, ref. 37). FasL activity was tested by apoptosis induction in Jurkat cells. Cell viability was assessed by MTT assay (Promega) and normalized to untreated controls.

Western blot analysis. Liver samples were lysed in PBS containing $300 \mathrm{mM}$ $\mathrm{NaCl}, 1 \%$ Triton X-100, and protease and phosphatase inhibitors (Roche
Applied Science) using a Dounce homogenizer (Wheaton). Cell lysates were separated by electrophoresis and transferred to nitrocellulose. JNK, phosphorylated JNK, and Bid (Cell Signaling Technology), Bim (SigmaAldrich), caspase 3, active caspase 3, caspase 8 (BD Biosciences - Pharmingen), and Fas (Santa Cruz Biotechnology Inc.) were detected by incubation of membranes with primary antibodies and corresponding horseradish peroxidase-labeled secondary antibodies. Signals were visualized by chemiluminescence. In some experiments, liver protein samples were treated with $0.1 \mathrm{U} / \mu \mathrm{l}$ of calf intestinal phosphatase (Promega) in $50 \mathrm{mM}$ Tris $\mathrm{pH}$ 9.3, $1 \mathrm{mM} \mathrm{MgCl}_{2}, 0.1 \mathrm{mM} \mathrm{ZnCl}_{2}$, and $1 \mathrm{mM}$ spermidine for 60 minutes at $37^{\circ} \mathrm{C}$, prior to detection of $\mathrm{Bim}_{\mathrm{EL}}$ by Western blot. 
Histology and immunohistochemistry. Liver tissue was fixed in 4\% paraformaldehyde in PBS and then embedded in paraffin. Five- $\mu \mathrm{m}$ sections were dewaxed with xylol and stained with $\mathrm{H} \& \mathrm{E}$ for histological assessment. For active caspase 3 immunohistochemistry, dewaxed sections were pretreated by boiling sections for 10 minutes in $10 \mathrm{mM}$ citrate, $\mathrm{pH}$ 6.0, in a pressure cooker to retrieve antigen, and unspecific binding was blocked with $5 \%$ goat serum, $1 \%$ casein, and $0.1 \%$ sodium azide in TBS. Liver sections were then stained with a rabbit anti-active caspase 3 antibody (1:100; Cell Signaling Technology) or irrelevant control antibody for 2 hours at room temperature and incubated with biotinylated goat anti-rabbit Ig antiserum (1:500; Dako) for 1 hour at room temperature. Thereafter, sections were incubated with a streptavidin-biotin/alkaline phosphatase complex (1:200 in TBS) (Dako) for 45 minutes. Finally, sections were developed with new fuchsin-naphthol AS-BI (Sigma-Aldrich) for 30 minutes, counterstained with hematoxylin, and mounted.

Statistics. Statistical differences were analyzed using unpaired 2-tailed Student's $t$ test. $P$ values less than 0.05 were considered significant.

\section{Acknowledgments}

The authors would like to thank Andreas Kappeler, the Brunner lab, and the Mueller lab for support; Seamus Martin, Andreas Strasser, Andreas Villunger, and Gottfried Baier for inspiring discussions; Adriano Fontana for the Neuro-2-FasL cell; Immunex for providing the TRAIL-deficient mice; and Andreas Strasser and Philippe Bouillet for the Bim-deficient mice. This work was supported by grants from COST 844, the Bernese Cancer League, the Swiss National Science Foundation, and Oncosuisse (to T. Brunner).

Received for publication December 20, 2005, and accepted in revised form July 11, 2006.

Address correspondence to: Thomas Brunner, Division of Immunopathology, Institute of Pathology, University of Bern, Murtenstrasse 31, 3010 Bern, Switzerland. Phone: 41-31-632-49-71; Fax: 41-31-381-87-64; E-mail: thomas.brunner@pathology.unibe.ch.
1. Hanahan, D., and Weinberg, R.A. 2000. The hallmarks of cancer. Cell. 100:57-70.

2. Evan, G.I., and Vousden, K.H. 2001. Proliferation, cell cycle and apoptosis in cancer. Nature. 411:342-348.

3. Wang, S., and El-Deiry, W.S. 2003. TRAIL and apoptosis induction by TNF-family death receptors. Oncogene. 22:8628-8633.

4. LeBlanc, H.N., and Ashkenazi, A. 2003. Apo2L/ TRAIL and its death and decoy receptors. Cell Death Differ. 10:66-75.

5. Walczak, H., et al. 1999. Tumoricidal activity of tumor necrosis factor-related apoptosis-inducing ligand in vivo. Nat. Med. 5:157-163.

6. Ashkenazi, A., et al. 1999. Safety and antitumor activity of recombinant soluble Apo2 ligand. J. Clin. Invest. 104:155-162.

7. Frese, S., Brunner, T., Gugger, M., Uduehi, A., and Schmid, R.A. 2002. Enhancement of Apo2L/TRAIL (tumor necrosis factor-related apoptosis-inducing ligand)-induced apoptosis in non-small cell lung cancer cell lines by chemotherapeutic agents without correlation to the expression level of cellular protease caspase- 8 inhibitory protein. J. Thorac. Cardiovasc. Surg. 123:168-174.

8. Ogasawara, J., et al. 1993. Lethal effect of the antiFas antibody in mice. Nature. 364:806-809.

9. Jo, M., et al. 2000. Apoptosis induced in normal human hepatocytes by tumor necrosis factor-related apoptosis-inducing ligand. Nat. Med. 6:564-567.

10. Zheng, S.J., Wang, P., Tsabary, G., and Chen, Y.H. 2004. Critical roles of TRAIL in hepatic cell death and hepatic inflammation. J. Clin. Invest. 113:58-64. doi:10.1172/JCI200419255.

11. Kondo, T., Suda, T., Fukuyama, H., Adachi, M., and Nagata, S. 1997. Essential roles of the Fas ligand in the development of hepatitis. Nat. Med. 3:409-413.

12. Kusters, S., et al. 1997. In vivo evidence for a functional role of both tumor necrosis factor (TNF) receptors and transmembrane TNF in experimental hepatitis. Eur. J. Immunol. 27:2870-2875.

13. Pinkoski, M.J., Brunner, T., Green, D.R., and Lin, T. 2000. Fas and Fas ligand in gut and liver. Am.J. Physiol. Gastrointest. Liver Physiol. 278:G354-G366.

14. Brunner, T., et al. 2003. Fas (CD95/Apo-1) ligand regulation in $\mathrm{T}$ cell homeostasis, cell-mediated cytotoxicity and immune pathology. Semin. Immunol. 15:167-176.

15. Lin, T., et al. 1998. Fas ligand-mediated killing by intestinal intraepithelial lymphocytes. Participation in intestinal graft-versus-host disease. J. Clin. Invest. 101:570-577.

16. Lawrence, D., et al. 2001. Differential hepatocyte toxicity of recombinant Apo2L/TRAIL versions. Nat. Med. 7:383-385.

17. Lin, Y., et al. 2000. The death domain kinase RIP is essential for TRAIL (Apo2L)-induced activation of IkappaB kinase and c-Jun $\mathrm{N}$-terminal kinase. $\mathrm{Mol}$. Cell. Biol. 20:6638-6645.

18. Baker, S.J., and Reddy, E.P. 1998. Modulation of life and death by the TNF receptor superfamily. Oncogene. 17:3261-3270.

19. Bennett, B.L., et al. 2001. SP600125, an anthrapyrazolone inhibitor of Jun N-terminal kinase. Proc. Natl. Acad. Sci. U. S. A. 98:13681-13686.

20. Putcha, G.V., et al. 2003. JNK-mediated BIM phosphorylation potentiates BAX-dependent apoptosis. Neuron. 38:899-914.

21. Puthalakath, H., Huang, D.C., O’Reilly, L.A., King, S.M., and Strasser, A. 1999. The proapoptotic activity of the Bcl-2 family member Bim is regulated by interaction with the dynein motor complex. $\mathrm{Mol}$. Cell. 3:287-296.

22. Chen, D., and Zhou, Q. 2004. Caspase cleavage of BimEL triggers a positive feedback amplification of apoptotic signaling. Proc. Natl. Acad. Sci. U. S. A. 101:1235-1240.

23. Bouillet, P., et al. 1999. Proapoptotic Bcl-2 relative Bim required for certain apoptotic responses, leukocyte homeostasis, and to preclude autoimmunity. Science. 286:1735-1738.

24. Corazza, N., et al. 2004. TRAIL and immunity: more than a license to kill tumor cells. Cell Death Differ. 11(Suppl. 2):S122-S125.

25. Seino, K., et al. 1997. Contribution of Fas ligand to $\mathrm{T}$ cell-mediated hepatic injury in mice. Gastroenterology. 113:1315-1322.

26. Yin, X., et al. 1999. Bid-deficient mice are resistant to Fas-induced hepatocellular apoptosis. Nature.
400:886-891

27. Li, H., Zhu, H., Xu, C.J., and Yuan, J. 1998. Cleavage of BID by caspase 8 mediates the mitochondrial damage in the Fas pathway of apoptosis. Cell. 94:491-501.

28. Gross, A., et al. 1999. Caspase cleaved BID targets mitochondria and is required for cytochrome $c$ release, while BCL-XL prevents this release but not tumor necrosis factor-R1/Fas death. J. Biol. Chem. 274:1156-1163.

29. Deng, Y., Lin, Y., and Wu, X. 2002. TRAIL-induced apoptosis requires Bax-dependent mitochondrial release of Smac/DIABLO. Genes Dev. 16:33-45.

30. Corazza, N., Brumatti, G., Jakob, S., Villunger, A., and Brunner, T. 2004. TRAIL and thymocyte apoptosis: not so deadly? Cell Death Differ. 11(Suppl. 2):S213-S215.

31. Takeda, K., et al. 2001. Involvement of tumor necrosis factor-related apoptosis-inducing ligand in surveillance of tumor metastasis by liver natural killer cells. Nat. Med. 7:94-100.

32. Cretney, E., et al. 2002. Increased susceptibility to tumor initiation and metastasis in TNF-related apoptosis-inducing ligand-deficient mice. J. Immunol. 168:1356-1361.

33. Smyth, M.J., et al. 2001. Tumor necrosis factorrelated apoptosis-inducing ligand (TRAIL) contributes to interferon gamma-dependent natural killer cell protection from tumor metastasis. J. Exp. Med. 193:661-670.

34. Spierings, D.C., et al. 2004. Tissue distribution of the death ligand TRAIL and its receptors. J. Histochem. Cytochem. 52:821-831.

35. Xu, Y., et al. 2003. Fc gamma Rs modulate cytotoxicity of anti-Fas antibodies: implications for agonistic antibody-based therapeutics. J. Immunol. 171:562-568.

36. Rensing-Ehl, A., et al. 1995. Local Fas /APO-1 (CD95) ligand-mediated tumor cell killing in vivo. Eur. J. Immunol. 25:2253-2258.

37. Holler, N., et al. 2003. Two adjacent trimeric Fas ligands are required for Fas signaling and formation of a death-inducing signaling complex. Mol. Cell. Biol. 23:1428-1440. 